\title{
Proposed Design of a Dissolution Apparatus for Vaginal Formulations Containing Probiotics
}

\author{
Vinita Kale ${ }^{1}$, Rashmi Trivedi, and Pratik Muley \\ Sharad Pawar College of Pharmacy, Wanadongri, Nagpur 441110, India
}

\begin{abstract}
In the pharmaceutical industry, dissolution testing is a very important tool in drug development and quality control. There is a need to establish the in vivo performance of products that contain probiotic agents for vaginal administration by developing in vitro methods of evaluation. In order to achieve a close in vitro-in vivo correlation for a tablet containing a probiotic agent for vaginal administration, the various parameters that must be considered when designing a dissolution apparatus include volume and composition of the dissolution medium, environmental conditions of the absorption site, and surface exposure of the tablet. The primary objective of the present investigation was the design of a dissolution apparatus that could simulate the vaginal environment for vaginal tablets containing a probiotic agent. The newly fabricated dissolution apparatus was found to be successful in minimizing the volume of the dissolution medium and in eliminating the risk of exposure of the vaginal formulation to agitation and sampling devices. Thus, the dissolution apparatus may prove to be of value for carrying out routine quality control tests.
\end{abstract}

\section{INTRODUCTION}

$\mathrm{n}$ the pharmaceutical industry, dissolution testing is a very important tool in drug development and quality control. Dissolution testing has recently been extended to a variety of novel or special dosage forms such as suspensions, orally disintegrating tablets, chewable tablets, vaginal tablets, chewing gums, transdermal patches, semisolid topical preparations, suppository implants, and injectable microparticulate formulations and liposomes (1).

Bacterial vaginosis, urinary tract infections, and yeast vaginitis affect an estimated one billion women each year. The only therapeutic treatment options are antimicrobial agents. In many cases, these are effective for treating the infection (2,3); however, recurrence, side effects, and secondary infections are frequent. Coinciding with infection is the disruption of commensal microflora in which Lactobacilli are also lost. The exogenous application of Lactiobacilli to the host as probiotic agents appears to offer an alternative management regimen to antimicrobial treatment and prophylaxis $(4,5)$.

The products that contain Lactobacillus include dairy products (yogurt, acidophilus milk) and commercially available Lactobacillus powders, tablets, and a few semisolid formulations.

The authors successfully developed the Lactobacillus (probiotic agent) tablets. These tablets are a special type of dosage form in that the released Lactobacilli attach to the vaginal membrane such that they colonize to form a protective environment against the vaginal pathogens.

To obtain a close in vitro-in vivo correlation for tablets containing a probiotic agent for vaginal administration, the parameters that must be considered (6) while designing or selecting a dissolution apparatus are:

'Corresponding author.
1. Volume of dissolution fluid.

2. Composition of dissolution fluid.

3. Environmental conditions of the absorption site.

4. Surface exposure of the tablet.

Taking this into account, the primary objective of the present investigation was the design of a dissolution apparatus that could simulate the vaginal environment for vaginal tablets containing a probiotic agent (Lactobacilli).

\section{MATERIALS AND METHODS \\ Materials}

The lyophilized Lactobacillus species was obtained as a gift from Uni Sankyo Ltd., Ciplun, India.

DeMan-Rogosa-Sharpe (MRS) medium was purchased from Himedia Laboratories Pvt. Ltd., Mumbai, India. Tablet excipients such as citric acid, sodium bicarbonate, and lactose were purchased from S.D. Fine Chemicals, Mumbai, India. All other chemicals were analytical grade and, where required, bacteriological grade.

\section{Tablet Preparation}

The tablets containing lyophilized Lactobacillus were prepared by a direct-compression technique. The lactobacillus and other ingredients of the formulation, shown in Table 1, were thoroughly mixed in a laboratory mortar. The mixture was then directly compressed on a single-punch tablet press (Manesty F-3) at a compression pressure of $3500 \mathrm{~kg} / \mathrm{cm}^{2}$ using a $9-\mathrm{mm}$ flat-faced punch. The batch size was 25 tablets.

\section{Dissolution Apparatus Design}

The design of the apparatus was a modified form of a Setnikar and Fantelli model (7), as shown in Figure 1. The dissolution apparatus was fabricated using neutral Corning ${ }^{\circledR}$ glass. The diameter of the outer tube was $4 \mathrm{~cm}$, inner tube was $1.5 \mathrm{~cm}$, and the total length of the 
Table 1. Formulation of Vaginal Tablet Containing Lyophilized Lactobacillus.

\begin{tabular}{lc}
\hline Ingredients & Weight $(\boldsymbol{m g})$ \\
\hline Lyophilized Lactobacillus & 300 \\
\hline Citric acid & 210 \\
\hline Sodium bicarbonate & 149 \\
\hline Lactose & 21 \\
\hline Total weight & 680 \\
\hline
\end{tabular}

apparatus was $10 \mathrm{~cm}$. The apparatus had two openings (like a condenser) for water circulation. The vaginal physiology was simulated by means of a $1.3-\mathrm{cm}$ diameter (inner) cellophane tube tied to both ends of the apparatus and partially filled with the MRS broth corrected to $\mathrm{pH} 4.5$ with an $85 \%(\mathrm{v} / \mathrm{v})$ solution of lactic acid. This apparatus was clamped in a slightly angular position $\left(30^{\circ}\right)$, as shown in Figure 1. Water at $37 \pm 1{ }^{\circ} \mathrm{C}$ was circulated through the openings of the apparatus (the directions are shown). Once the temperature of the circulating water was equilibrated, the tablet containing probiotic agent was dropped into the cellophane tube; after $10 \mathrm{~min}, 5 \mathrm{~mL}$ of MRS broth was added over the tablet. A container of sterile water was placed under the discharge outlet to collect the discharged liquid. The disintegration time was noted. The test was carried out in 10 replicates.

\section{In Vitro Measurement of Probiotic}

The probiotic agent present was tested using the method described previously by the author (8). The Lactobacilli were grown in pre-sterilized MRS broth medium by inoculating $9.5 \mathrm{~mL}$ of medium with $0.5 \mathrm{~mL}$ of discharge fluid. The probiotic species was grown in the culture tubes, incubated at $37^{\circ} \mathrm{C}$ in an atmosphere of $5 \%$

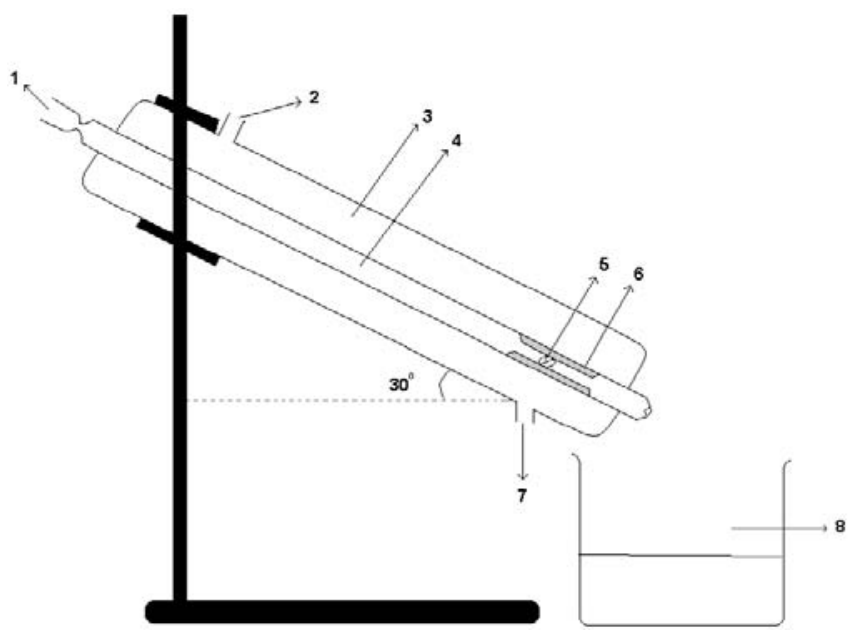

Figure 1. Design of a dissolution apparatus for vaginal formulations containing probiotics.

1- Inlet for MRS medium ( $5 \mathrm{~mL}), \mathbf{2}$-Inlet for warm water $\left(37^{\circ} \mathrm{C}\right)$, 3-Outer tube $(4 \mathrm{~cm}), 4$ - Inner Tube $(1.5 \mathrm{~cm}), 5$-Vaginal tablet, 6-Cellophane membrane, 7- Outlet for warm water, 8-Collecting vessel.
$\mathrm{CO}_{2}$ and $95 \%$ air for $24 \mathrm{~h}$. After the prescribed period of incubation, growth was measured on an optical density monitoring system (Toshniwal, India). This experiment was performed in triplicate.

\section{RESULTS AND DISCUSSION}

The vaginal tablets containing Lactobacillus were prepared successfully (Tables 1 and 2). The dissolution test measures the amount of active released from the formulation as a function of time. The modified dissolution apparatus was designed to mimic the in vivo conditions of the vaginal environment to study the release of probiotic from the vaginal tablet. The dissolution time for the tablets in the designed experiment was found to be $22 \pm 0.43 \mathrm{~min}$ $(n=6)$. The proposed method is essentially an improvement over the Setnikar et al. (7) apparatus with a modification of dissolution fluid volume (considering vaginal compartment) from 500 to $5 \mathrm{~mL}$ and a change in the apparatus position from vertical to angular $\left(60^{\circ}\right)$ so as to mimic the in vivo conditions during the study. The vaginal fluid is simulated by the addition of $80 \%(\mathrm{v} / \mathrm{v})$ lactic acid to MRS medium to attain a pH of $4.5(9,10)$. Similarly, the composition of MRS broth as dissolution fluid provides a natural growth environment for the probiotic agent (Lactobacillus).

In the compendial method for determining the dissolution time for a vaginal formulation, the formulation is hung into a beaker containing dissolution fluid that is stirred with a paddle rotator. The drawbacks of this method are that the volume of dissolution fluid is large (500-900 mL), there is no control of the mass-medium interface ratio, and it cannot simulate the in vivo environment (11).

In order to control the mass-medium ratio, several means have been employed that include a wire-mesh basket (12) or a membrane to separate the sample chamber from the reservoir (13). In these models, also, the volume of dissolution fluid is large and does not match the vaginal discharge volume.

The flow-cell apparatus that holds the sample in place with cotton or wire screen or glass beads (11) has a disadvantage in that the formulation is held in an upright position in such a way that the released probiotic agent leaks out in the discharged fluid and can only adhere to minimal membrane surface.

The dialysis bag method developed by Setnikar and Fantellis (7) almost simulates the environment for a rectal formulation. So this model was modified to design a suitable apparatus for dissolution testing of a vaginal formulation containing a probiotic agent.

Table 2. Physical Characterization of a Vaginal Tablet Containing Lyophilized Lactobacillus.

\begin{tabular}{ll}
\hline Parameter & \multicolumn{1}{c}{ Observation } \\
\hline Hardness & $4 \pm 0.24 \mathrm{~kg} / \mathrm{cm}^{2}(\mathrm{n}=6)$ \\
\hline$\%$ Friability & $0.83 \pm 0.1(\mathrm{n}=6)$ \\
\hline Disintegration Time & $22 \pm 0.43 \min (\mathrm{n}=6)$ \\
\hline
\end{tabular}


After $24 \mathrm{~h}$ of incubation, Lactobacillus species exhibited growth with an optical density between 0.06 and 0.09 . This estimated number (less than $10^{2}-10^{3} \mathrm{cfu} / \mathrm{mL}$ ) indicates that very little quantity of discharged fluid containing Lactobacilli leaked from the administration site (dissolution apparatus). This indicates that the developed assembly was satisfactory in simulating the application site for vaginal formulations.

\section{CONCLUSION}

In summary, this study concludes that for vaginal formulations containing probiotic agents, full exposure to and maintenance of continuous body temperature resulted in the complete dissolution and release of the probiotic. In this investigation with a newly modified dissolution apparatus, in vivo conditions including vaginal discharge volume were closely simulated. The advantages of the apparatus are minimization of dissolution fluid volume and elimination of the risk of exposure of the vaginal formulation to agitation and sampling devices. The dissolution time and growth of the probiotic agent could be accurately measured. Thus, it can be concluded that the newly fabricated dissolution apparatus is a very promising method and can be adopted for routine quality control testing.

\section{References}

1. Siewert, M.; Dressman, J.; Brown, C. K.; Shah, V.P. FIP/ AAPS Guidelines to dissolution/in vitro release testing of novel/special dosage forms. AAPS PharmSciTech 2003, 4 (1), Article 7.

2. Ritter, W.; Patzschke, K.; Krause, U.; Stettendorf, S. Pharmacokinetic fundamentals of vaginal treatment with clotrimazole. Chemotherapy 1982, 28 (Suppl. 1), 37-42.
3. Deshpande, A. A.; Rhodes, C.T.; Danish, M. Intravaginal drug delivery. Drug Dev. Ind. Pharm. 1992, 18, 1225-1279.

4. Hallen, A.; Pahlson, C. Treatment of bacterial vaginosis with lactobacilli. Sex Trans. Dis. 1992, 19, 146-148.

5. Reid, G. Probiotics for urogenital health. Nutr. Clin. Care. 2002, 5 (1), 3-8.

6. Ceschel, G. C; Maffei, P.; Lambardi Borgia, S.; Ronchi, C.; Rossi, S. Development of a mucoadhesive dosage form for vaginal administration. Drug Dev. Ind. Pharm. 2001, 27 (6), 541-547.

7. Setnikar, I.; Fantelli, S. Liquefaction time of rectal suppositories. J. Pharm. Sci. 1962, 51 (6), 566-571.

8. Kale, V.; Dabre, R.; Bhusari, K. A new approach to the formulation of lactobacilli used as probiotic and anti diarrheal agent. Indian Drugs 1998, 35 (5), 281-285.

9. Nowroozi, J.; Mirzaii, M. Study of lactobacillus as probiotic bacteria. Iranian J. Pub. Health 2004, 33 (22), 1-7.

10. Ceschel, G. C.; Rossi, S. Development of mucoadhesive dosage form for vaginal administration. Drug Dev. Ind. Phar. 2001, 27 (6), 541-547.

11. Pandit, J. K.; Choudhary, P. K.; Mishra, B. Modified bead-bed continuous flow dissolution apparatus for in vitro evaluation of suppository. Indian Pharmacist 1982, 32 (5), 132-134.

12. Babar, A.; Bellete, T.; Pakogiannis, F. M. Ketoprofen suppository dosage forms: In vitro release and in vivo absorption studies in rabbits. Drug Dev. Ind. Pharm. 1999, 25 (2), 241-245.

13. Azechi, Y.; Ishikawa, K.; Takahashi, K. Sustained release of diclofenac from polymer containing suppository and mechanism involved. Drug. Dev. Ind. Pharm. 2000, 26 (11), 1177-1183. 